\title{
PARAMETRIZAÇÃO DE ABÓBADAS EM CASCAS EM CONCRETO ARMADO COM VISTAS AOS ÍNDICES DE CONSUMO DE MATERIAIS 1
}

\section{PERFORMANCE RATIOS OF PARAMETRIC AND MODULAR CONCRETE SHELL VAULTS STRUCTURES}

\author{
Lucio José Muniz \\ Universidade Federal da Bahia (UFBA) \\ lucio.jose@ufba.br \\ Felipe Tavares da Silva \\ Universidade Federal da Bahia (UFBA) \\ felipe.tavares@ufba.br
}

\begin{abstract}
Resumo
A construção de abóbadas em cascas de concreto armado para coberturas implica em elevados custos em razão da utilização de grandes quantidades de materiais. Este estudo visa a identificação do desempenho de abóbadas em casca em geometria de arco parabólico e sua consequente espessura pré-dimensionada. A partir da parametrização deste sistema, foi construído um banco de dados contendo as taxas de armadura por área coberta pela abóbada, gerando a forma a partir de um algoritmo generativo, e obtendo a quantidade de armadura necessária através de um software de análise estrutural. Após a criação do banco de dados foi ajustada uma função que relaciona os parâmetros da geometria da abóbada com a o consumo de aço por área coberta. Os parâmetros geométricos considerados foram: o vão, a altura do arco parabólico, a resistência do concreto e a espessura da casca. Esta função pode ser útil para avaliar, junto ao algoritmo generativo de estruturas, o consumo de aço por área coberta, fornecendo assim o quantitativo de armadura nas fases iniciais de concepção estrutural deste sistema.
\end{abstract}

Palavras-chave: Abóbada parametrizada. Elementos finitos. Concreto armado. Taxa de armadura.

\begin{abstract}
The construction of reinforced concrete vaults for roofing shells implies high costs due to the use of large quantities of materials. This study aims to identify the performance of shell vaults on a parabolic arch geometry and the consequent pre-sized thickness. From a parametric formulation of this system, a database was constructed containing the reinforcement rates per area covered by the vault, generating the shape from a generative algorithm, and obtaining the amount of required reinforcement by a structural analysis software. After creating the database was set a function that fits the reinforcement ratio from the geometry parameters of the vault. The geometrical parameters considered was: the span of the arch, the height of the parabolic arch, the concrete strength and the thickness of the shell. This function can be useful to evaluate along a structural generative algorithm the reinforcement steel consumption by covered area, thereby providing the reinforcement quantitative in the initial stages of the structural design of this system.
\end{abstract}

Keywords: Parametric vault. Finite element. Reinforced concrete; Reinforcement ratio. 


\section{INTRODUÇÃO}

Um grande número de edificações em casca de concreto armado foi utilizado em contextos arquitetônicos no Brasil e no mundo. Particularmente na geometria de abóbada de berço, a Igreja da Pampulha, em Belo Horizonte, pode ser tomada como exemplo de uma abóbada regular e parabólica. Entretanto, poucas são as informações sobre os indicadores de consumo de material destes sistemas estruturais.

REBELO (2011) apresenta de forma generalizada, através de ábacos traduzidos de CORKILL (1969), as relações entre espessura e vão de abóbadas em casca de concreto armado. Entretanto estes ábacos são referentes a concretos elaborados no século passado com características e resistências inferiores aos utilizados nos dias de hoje. Os mais recentes aprimoramentos incorporados aos materiais, sejam relacionados ao desenvolvimento de novos materiais, ou na composição de aditivos do cimento e do concreto, que refletem em alterações no desempenho do concreto armado, geram novas demandas de pesquisas para uma nova avaliação do desempenho destas estruturas.

Não foram encontrados na literatura relatos de índices de consumo de material para abóbadas em concreto. Contudo, OLIVEIRA et al. (2013) propõem indicadores de consumo de concreto, aço e formas de concreto para sistemas de piso em concreto armado. Estes autores relataram taxas de consumo para sistemas de piso com lajes maciças e nervuradas apoiadas em vigas e do tipo cogumelo com vãos entre $4,0 \mathrm{~m}$ e $5,5 \mathrm{~m}$, relatando valores da taxa de concreto entre 0,15 e $0,21 \mathrm{~m}^{3} / \mathrm{m}^{2}$, e taxa de aço ente 8,49 e $19,89 \mathrm{Kg} / \mathrm{m}^{2}$ de planta. Em outro estudo conduzido por PEREIRA et al.(2013), também em relação a sistemas de lajes com vãos entre $3,0 \mathrm{~m}$ e $4,8 \mathrm{~m}$, foi relatado valores de taxa de concreto entre 0,11 e $0,19 \mathrm{~m}^{3} / \mathrm{m}^{2}$ e taxas de armadura entre 6,59 e $12,65 \mathrm{Kg} / \mathrm{m}^{2}$.

TAVARES (2006) listou taxas de quantidades das estruturas de concreto relatando valores que variam de $0,05 \mathrm{~m}^{3} / \mathrm{m}^{2}$ de concreto para estruturas até 4 pavimentos a $0,22 \mathrm{~m}^{3} / \mathrm{m}^{2}$ para estruturas de 11 a 30 pavimentos. As taxas de aço relacionadas foram de $4,75 \mathrm{Kg} / \mathrm{m}^{2}$ a 28,6 $\mathrm{Kg} / \mathrm{m}^{2}$ e $95 \mathrm{Kg} / \mathrm{m}^{3}$ a $130 \mathrm{Kg} / \mathrm{m}^{3}$.

A variação da quantidade de concreto e de aço na composição do concreto armado reflete no custo da obra, na quantidade de energia incorporada e na emissão de $\mathrm{CO}_{2}$. Fatores estes que devem ser analisados, pois são determinantes em relação à viabilidade técnica e econômica e no impacto causado ao meio ambiente.

TAVARES (2006) lista valores de energia incorporada e emissão de $\mathrm{CO}_{2}$ relativos ao consumo de concreto e aço das armaduras. Foi relatado por este autor que para cada $\mathrm{m}^{3}$ de concreto são incorporados $2760 \mathrm{MJ}$ de energia enquanto que cada $\mathrm{kg}$ de aço de armadura possui 30 $\mathrm{MJ}$ de energia incorporada ao processo. Além disto, ele relata que para cada $\mathrm{m}^{3}$ de concreto são emitidos $45 \mathrm{Kg}$ de $\mathrm{CO}_{2}$, e para cada $\mathrm{kg}$ de aço são emitidos $2,46 \mathrm{Kg}$ de $\mathrm{CO}_{2}$.

Por outro lado, a base de dados espanhola do ITEC (Instituto de Tecnología de La Construccíon) relata que para cada $\mathrm{m}^{3}$ de concreto são incorporados na sua produção 1462 $\mathrm{MJ}$ de energia e $271 \mathrm{Kg} \mathrm{CO}$, enquanto que para cada $\mathrm{kg}$ de armadura são incorporados 37 $\mathrm{MJ}$ e energia e $3,00 \mathrm{Kg}$ de $\mathrm{CO}_{2}$.

GRAF e TAVARES (2010) obtiveram para uma edificação modelo, térreo de pequeno porte $\left(184 \mathrm{~m}^{2}\right)$, um valor de $1427 \mathrm{MJ}$ de energia incorporada por $\mathrm{m}^{2}$ da planta da estrutura.

OLIVEIRA et al.(2013) relatam, nas estruturas em concreto multipavimentos estudadas, uma taxa de 1130 a $1700 \mathrm{MJ} / \mathrm{m}^{2}$ de estrutura e uma taxa de 115,64 a $134,72 \mathrm{Kg} \mathrm{CO} / \mathrm{m}^{2}$ de estrutura.

Com base nestes dados de sistemas aporticados pode-se fazer um comparativo relativo dos índices encontrados para abóbadas de casca em concreto armado de forma regular e parabólica. 
Um banco de dados, contendo informações de taxas de consumo de materiais para a construção de abóbadas moduladas em casca de concreto representa uma fonte de subsídios que auxiliarão arquitetos e engenheiros na concepção destas estruturas arquitetônicas, sobretudo no que diz respeito à verificação da viabilidade técnica e econômica da sua escolha. A identificação dos índices de volume de concreto e peso de aço na fabricação de sistemas em abóbadas em cascas, bem como uma comparação com os sistemas já construídos em geometrias similares, dará uma visão global sobre as combinações de geometrias e resistências do material, bem como uma reflexão sobre geometrias ótimas.

A construção deste Banco de Dados foi realizada em duas etapas. A primeira consistiu na geração do pré-dimensionamento parametrizado das estruturas de abóbadas em casca através de expressões matemáticas de pré-dimensionamento em função da espessura da casca, da resistência do concreto e da relação Flecha/Vão. Na segunda etapa, as informações obtidas foram organizadas em tabelas contendo as dimensões dos protótipos a serem calculados, realizando os cálculos de deslocamento e taxa de armadura através do software de análise estrutural Autodesk Robot.

\section{METODOLOGIA}

Para a geração da geometria da abóbada foi utilizada a expressão matemática que relaciona o vão e a flecha do arco parabólico dada pela Equação 1, onde o comprimento do arco é dado pela Equação 2.

$$
\begin{aligned}
& G(x)=\left(\frac{-4 \cdot f}{L^{2}}\right) \cdot x^{2}+\left(\frac{4 \cdot f}{L}\right) \cdot x \\
& C_{\text {arco }}=\frac{L^{2}}{16 \cdot f} \cdot\left\{\frac{8 \cdot f}{L} \cdot \sqrt{1+\left(\frac{4 \cdot f}{L}\right)^{2}}+\ln \left[\frac{4 \cdot f}{L}+\sqrt{1+\left(\frac{4 \cdot f}{L}\right)^{2}}\right]-\ln \left[\frac{-4 \cdot f}{L}+\sqrt{1+\left(\frac{4 \cdot f}{L}\right)^{2}}\right]\right\}
\end{aligned}
$$

Nas expressões [1] e [2], $f$ é a flecha do arco parabólico, $L$ o vão do arco, $G(x)$ a função que define a geometria do arco e $C_{\text {arco }}$ o comprimento curvo do arco.

Além da forma geométrica do arco da abóbada, foi também parametrizada a relação entre a espessura da casca, as dimensões da flecha e do vão e a resistência do concreto. Para isto, foram determinados o esforço normal e o momento fletor de projeto a partir da solução simplificada de um arco isostático tri-articulado, dados pelas expressões [3] e [4], sendo este arco submetido apenas à carga gravitacional.

$$
\begin{aligned}
& N_{d}=\frac{1,4 \cdot\left(0,0025 \cdot d \cdot C_{\text {arco }}+q_{s c} \cdot L\right)}{-10,38 \cdot\left(\frac{f}{L}\right)^{4}+29,56 \cdot\left(\frac{f}{L}\right)^{3}-29,79 \cdot\left(\frac{f}{L}\right)^{2}+11,22 \cdot\left(\frac{f}{L}\right)-0,12} \\
& M_{d}=\frac{1,4 \cdot\left(0,0025 \cdot d \cdot C_{\text {arco }} \cdot L+q_{s c} \cdot L^{2}\right)}{12350 \cdot \exp \left(-17,93 \cdot \frac{f}{L}\right)+534 \cdot \exp \left(-2,61 \cdot \frac{f}{L}\right)}
\end{aligned}
$$

De forma simplificada, foi considerado o esforço normal resistente na base do arco como sendo uma compressão simples [5], considerando uma taxa de armadura de $0,2 \%$, e o momento fletor resistente no primeiro quarto do vão, considerando o caso de flexão simples [6] (NBR6118, 2003). 
$N_{d R E S}=\left(7,14 \cdot f_{c k}+8,69\right) \cdot d$

$$
M_{d R E S}=0,0129 \cdot f_{c k} \cdot d^{2}
$$

Nas expressões de [3] a [6], $d$ é a espessura da casca em centímetros, $q_{s c}$ a sobrecarga sobre a casca em $\mathrm{KN} / \mathrm{m}^{2}$, comprimento do vão $L$ e do arco $C_{\text {arco }}$ em metros, e $f_{c k}$ a resistência característica do concreto em MPa.

A geração da forma pré-dimensionada da estrutura para cada incremento de vão foi dada pela satisfação da condição de capacidade resistente da peça, satisfazendo a condição de que a solicitação de projeto deve ser inferior à resistência de projeto: $S_{d} \leq R_{d}$ (NBR6118, 2003). Assim, foram obtidas espessuras mínimas para cada incremento de vão, contanto que fossem satisfeitas as condições do esforço normal e momento fletor simultaneamente. As expressões propostas de pré-dimensionamento das abóbadas em casca de concreto são dadas pelas equações [7] e [8], obtidas pelas substituições da equação [5] na [3] e [6] na [4].

$$
\begin{aligned}
& d_{E N} \geq \frac{1,4 \cdot\left(0,0025 \cdot d \cdot C_{\text {arco }}+q_{s c} \cdot L\right)}{\left[-10,38 \cdot\left(\frac{f}{L}\right)^{4}+29,56 \cdot\left(\frac{f}{L}\right)^{3}-29,79 \cdot\left(\frac{f}{L}\right)^{2}+11,22 \cdot\left(\frac{f}{L}\right)-0,12\right] \cdot\left(7,14 \cdot f_{c k}+8,69\right)} \\
& d_{M F} \geq \frac{1,4 \cdot\left(0,0025 \cdot d \cdot C_{\text {arco }} \cdot L+q_{s c} \cdot L^{2}\right)}{\left[12350 \cdot \exp \left(-17,93 \cdot \frac{f}{L}\right)+534 \cdot \exp \left(-2,61 \cdot \frac{f}{L}\right)\right] \cdot 0,0129 \cdot f_{c k}}
\end{aligned}
$$

O maior dos valores obtidos pelas expressões [7] e [8] é a espessura mínima para a casca.

O domínio definido para estes parâmetros foram valores que variam de 10 a 50 metros para o vão $L$, com intervalos de 5 metros; variações de 0,1 a 1,0 para a relação $(f / L)$, com incrementos de 0,1 ; Resistência à compressão $\left(f_{c k}\right)$ com valores de $20 \mathrm{MPa}$ a $50 \mathrm{MPa}$, considerando incrementos de $5 \mathrm{MPa}$.

De posse destas informações, foram implementados os modelos gerados no Autodesk Robot através desta combinação de parâmetros de entrada, realizadas simulações, submetendo a estrutura aos esforços de peso próprio adicionando ao sistema uma sobrecarga de $2,5 \mathrm{KN} / \mathrm{m}^{2}$, representativa para eventuais solicitações. Com isto, foram obtidos, por intermédio de gráficos, o deslocamento máximo e a taxa de armadura. Estas informações foram utilizadas para a obtenção dos índices de consumo de concreto por área coberta $\left(\mathrm{m}^{3} / \mathrm{m}^{2}\right)$, a taxa total de aço por área coberta $\left(\mathrm{kg} / \mathrm{m}^{2}\right)$, a taxa de aço por volume de concreto $\left(\mathrm{kg} / \mathrm{m}^{3}\right)$ e a taxa de forma por área coberta $\left(\mathrm{m}^{2} / \mathrm{m}^{2}\right)$.

\section{RESULTADOS}

A geometria de cada protótipo analisado foi obtida por extrusão do arco parabólico e em seguida gerada a malha de elementos finitos de casca no ambiente do Autodesk Robot, constituindo assim o modelo estrutural 3d da abóbada, como ilustra a Figura 1. 


\section{TIC2015}

Figura 1 - Modelo estrutural de uma das cascas protótipo no Autodesk Robot

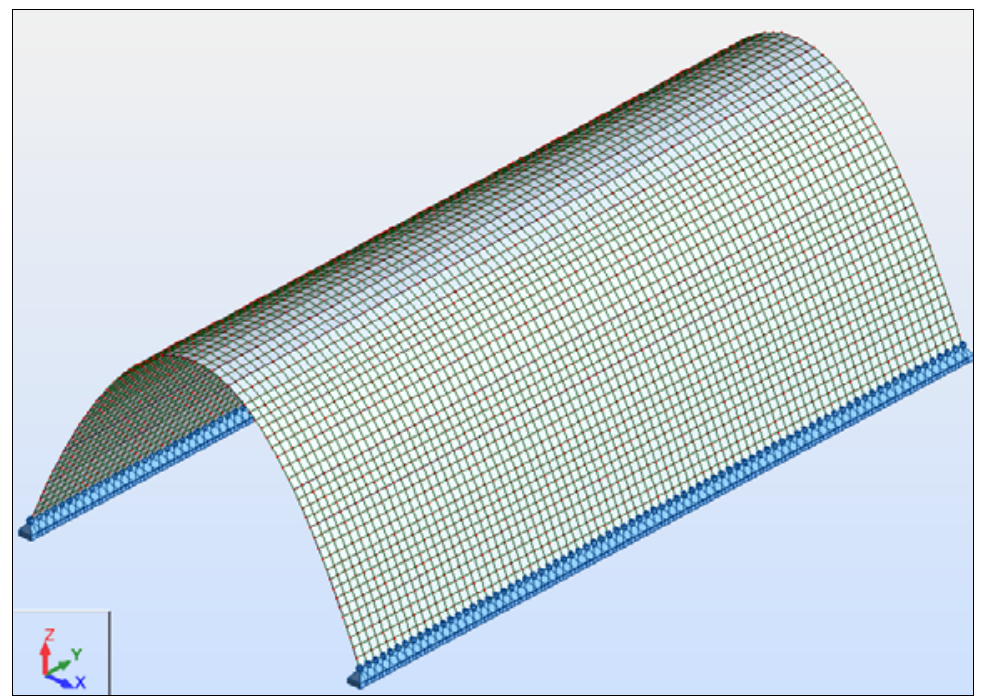

Fonte: Preparada pelos autores.

Nestes modelos de abóbadas em cascas foram considerados elementos quadrilaterais de 4 nós e todos os cálculos no ambiente do Robot são realizados pelo método dos elementos finitos. Na combinação de carga para a determinação da área de aço necessária, considerouse uma majoração das cargas de 1,4 e para verificação dos deslocamentos considerou-se um fator de 1,0. Após a aplicação das cargas de peso próprio e sobrecarga no modelo estrutural, para cada protótipo foram obtidas os deslocamentos estruturais e as taxas de armaduras nas duas faces superficiais da casca. Os resultados de deslocamento e cálculo de armadura de uma determinada configuração de abóbada podem ser visualizados nas Figuras 2, 3 e 4, respectivamente.

Figura 2 - Deslocamentos estruturais obtidos do modelo estrutural

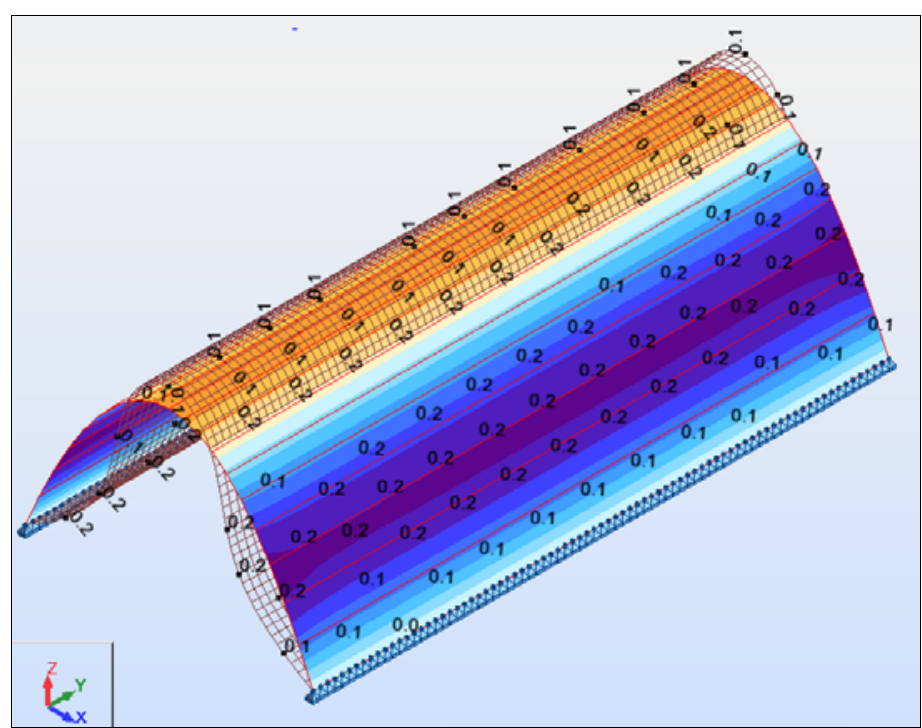

Fonte: preparada pelos autores 
Figura 3 - Área de aço necessária na face externa da casca de concreto

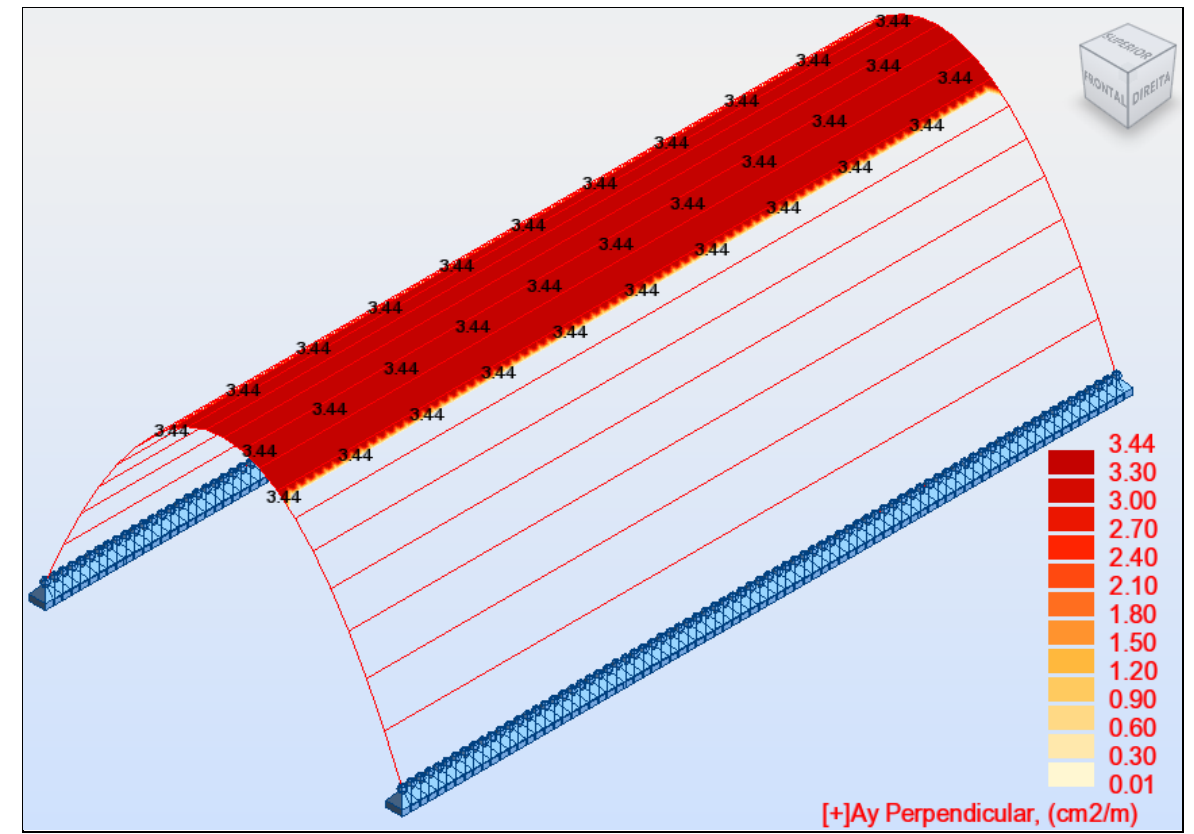

Fonte: preparada pelos autores

Figura 4 - Área de aço necessária na face interna da casca de concreto

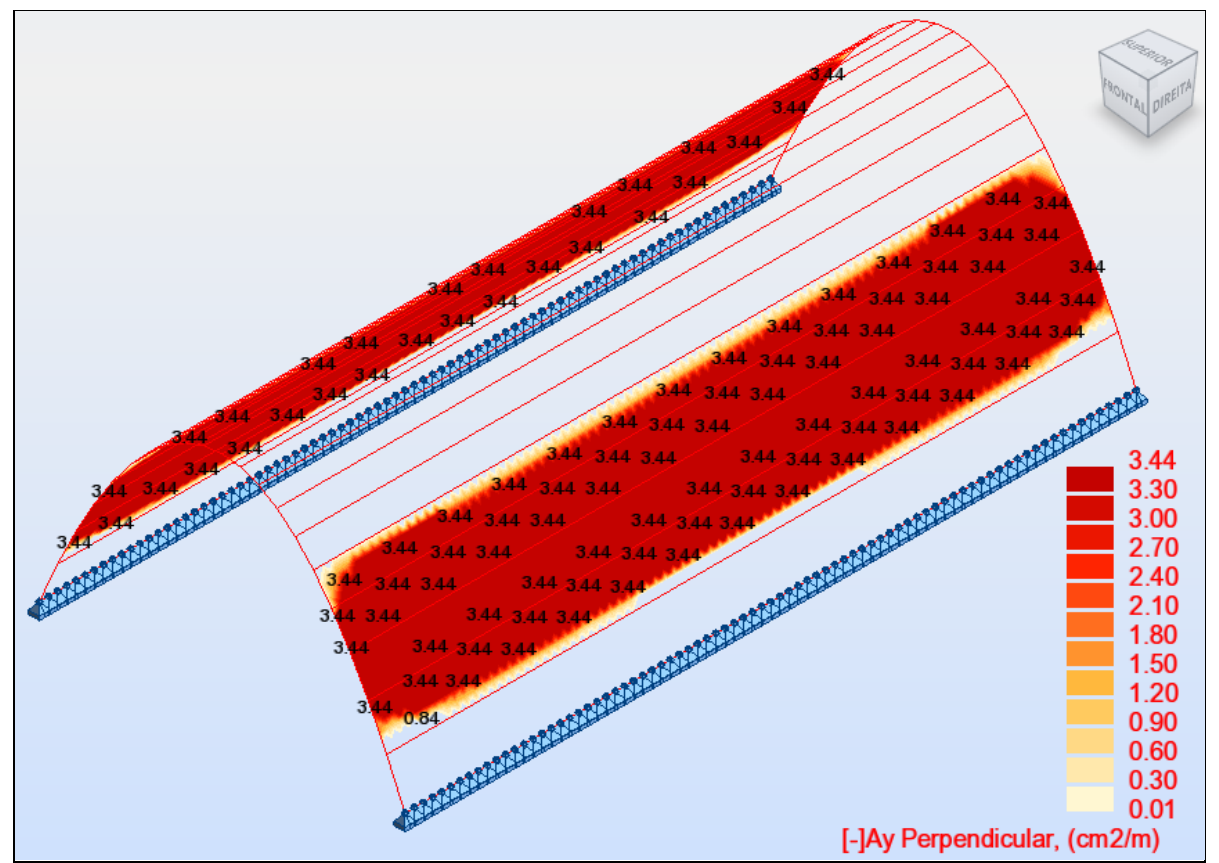

Fonte: preparada pelos autores

Para cada caso, foi considerada no quantitativo da armadura a maior seção de aço de uma das faces e generalizada para toda a casca de forma simétrica.

Todos os casos em que o programa de análise estrutural acusou armadura excessiva ou deslocamento excessivo foram eliminados do domínio de análise. 
Figura 5 - Ábaco com a relação entre vão e espessura da abóbada para o domínio obtido

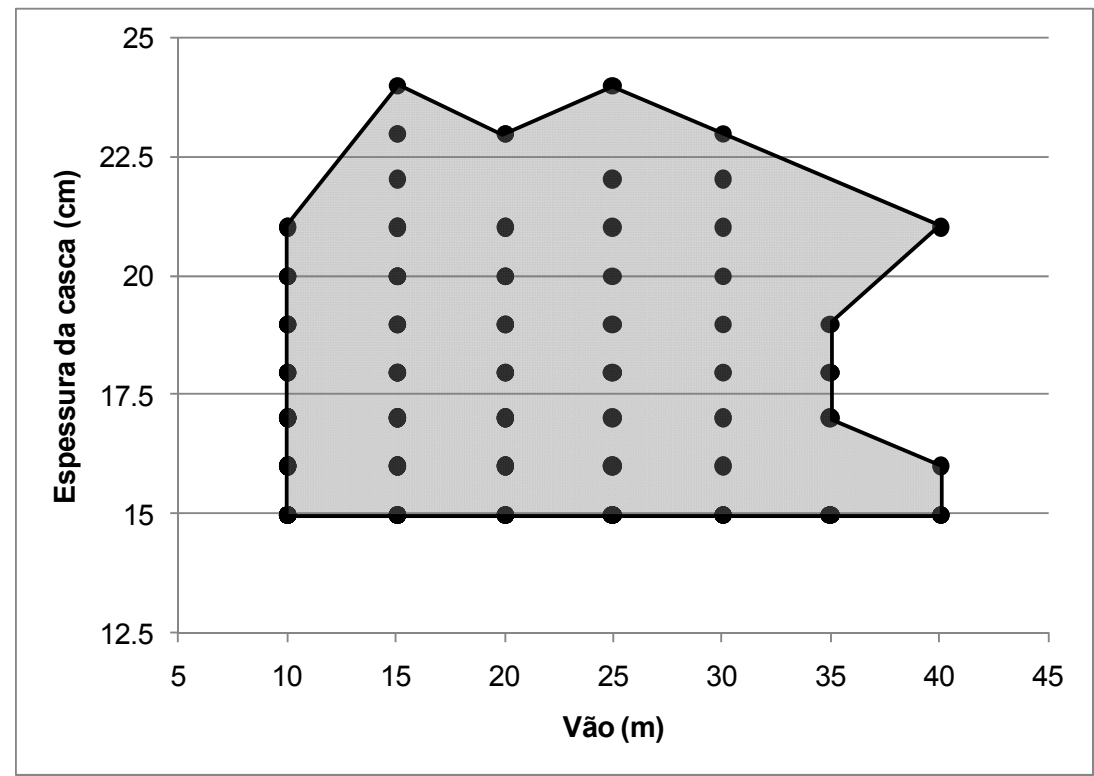

Fonte: preparada pelos autores

Na Figura 5 pode ser observada a ilustração do domínio válido obtido por este processamento na análise estrutural, evidenciando a relação entre vão e espessura da casca.

Para cada configuração de vão $L$, relação entre flecha e vão $f / L$ e resistência do concreto $f_{c k}$, foram calculadas as taxas de armadura. Após realizar o cálculo sobre todo o domínio definido foi possível ajustar funções ([9] a [12]) que definem a taxa de armadura da abóbada em casca de concreto armado em função destes três parâmetros de entrada.

$$
\begin{aligned}
& \text { TCm2 }=0,106+(0,00198) \cdot L+(0,342) \cdot f / L+(-0,00112) \cdot f_{c k} \\
& \text { TAm2 }=7,35+(0,016) \cdot L+(16,95) \cdot f / L+(0,0025) \cdot f_{c k} \\
& \text { TAm3 }=70,82+(-0,64) \cdot L+(-18,73) \cdot f / L+(0,293) \cdot f_{c k} \\
& \text { TFm2 }=0,79+(0,00097) \cdot L+(1,39) \cdot f / L+(0,00039) \cdot f_{c k}
\end{aligned}
$$

Na expressão [9] está definida a taxa de consumo de concreto por área de planta da abóbada $T C m 2$ em $\mathrm{m}^{3}$ de concreto por $\mathrm{m}^{2}$ de área coberta, também conhecida como espessura fictícia. $\mathrm{Na}$ expressão [10] está definida a taxa de armadura em relação à área de planta coberta pela abóbada TAm2, dada em $\mathrm{kg} / \mathrm{m}^{2}$. Na expressão [11] está definida a taxa de armadura em relação ao volume de concreto $T A m 3$, dada em $\mathrm{kg} / \mathrm{m}^{3}$. Na expressão [12] está definida a taxa de forma em relação à área de planta coberta, dada $\mathrm{em} \mathrm{m}^{2} / \mathrm{m}^{2}$.

As unidades das variáveis nas expressões de [9] a [12], sendo o vão $L$ em metros, razão da flecha pelo vão $f / L$ adimensional e a resistência do concreto $f_{c k}$ em MPa.

Estas expressões que fornecem o consumo de materiais para a construção da abóbada em concreto armado são válidas apenas no domínio explicitado pela ilustração da figura 6 e podem ser utilizadas em algoritmos generativos deste sistema para prever o consumo de materiais ainda na fase de concepção deste sistema. 
Figura 6 - Variação da taxa de concreto das cascas em estudo (fck $=30 \mathrm{MPa})$

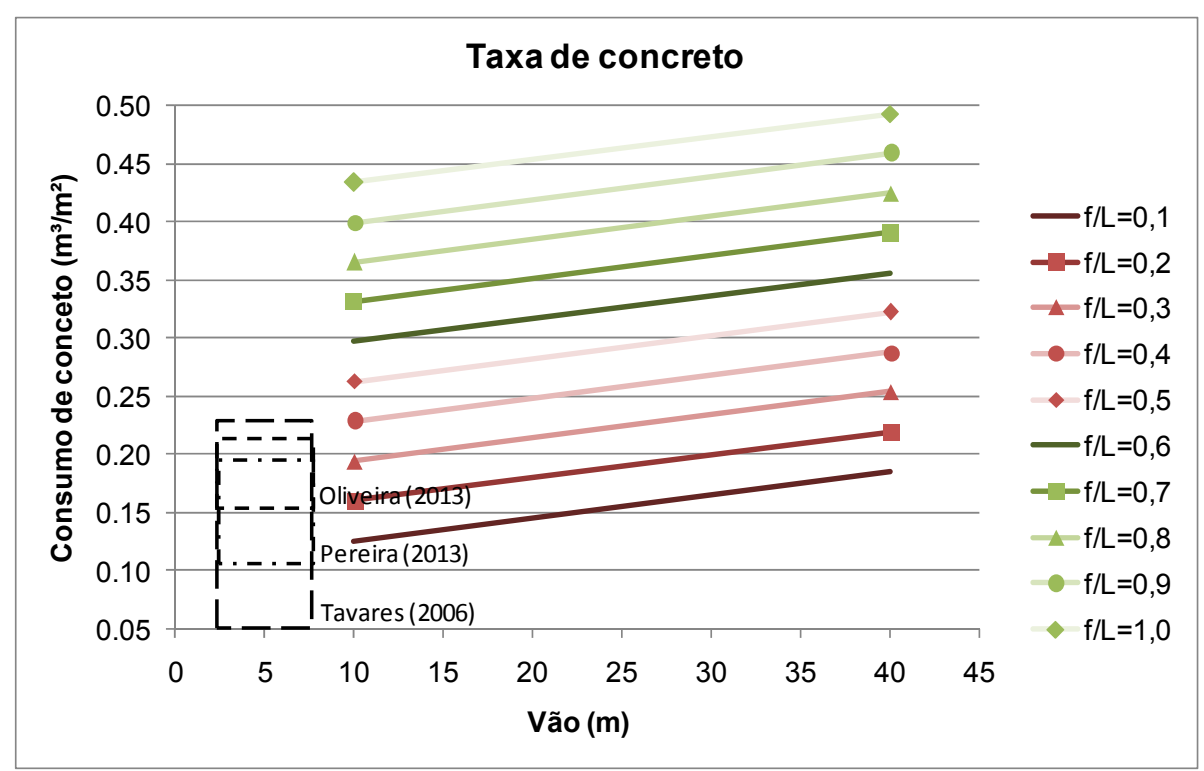

Fonte: preparada pelos autores

Figura 7 - Variação da taxa de aço por área coberta (fck = $30 \mathrm{MPa})$

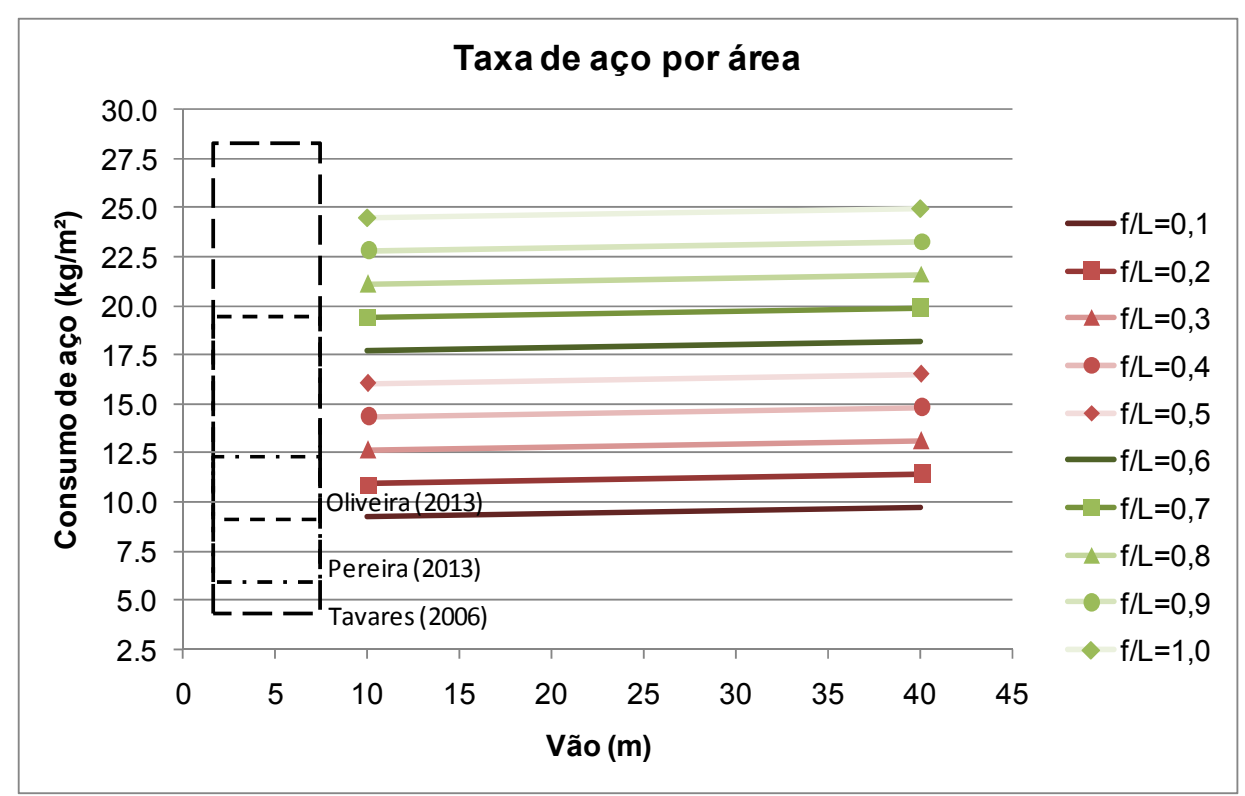

Fonte: preparada pelos autores 
Figura 8 - Variação da taxa de aço por volume de concreto (fck = $30 \mathrm{MPa}$ )

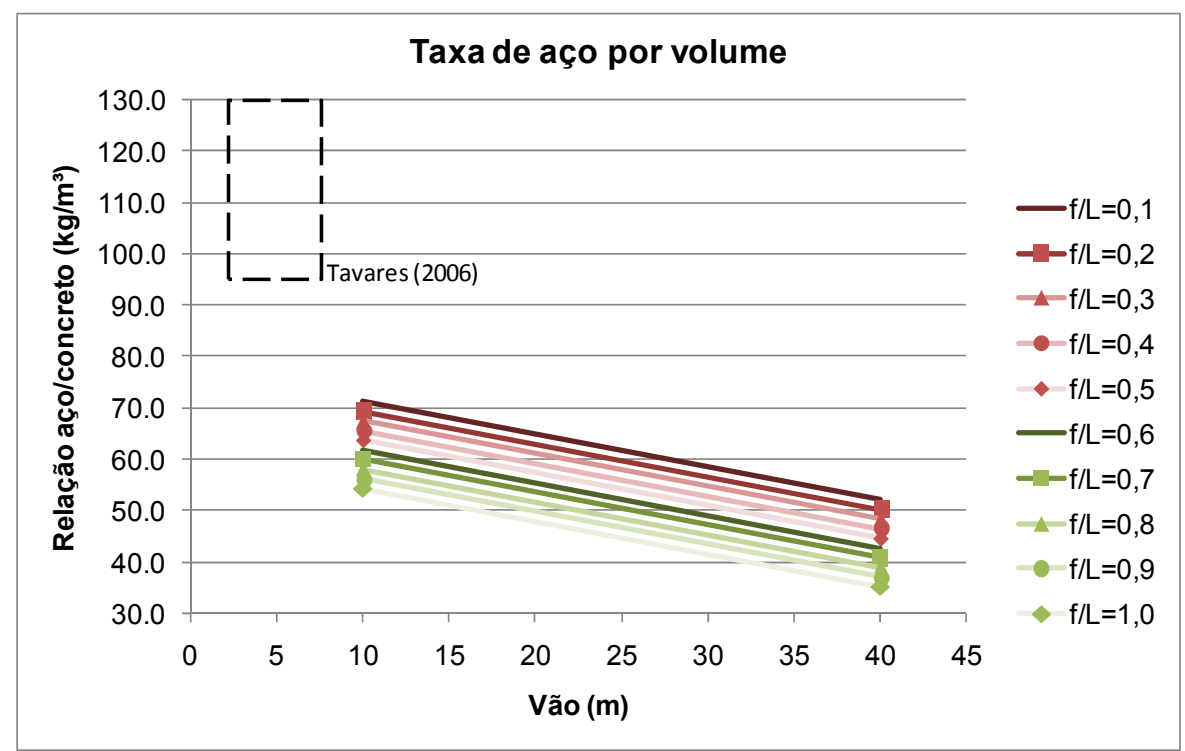

Fonte: preparada pelos autores

Nas Figuras de 6 a 8 estão ilustrados os gráficos obtidos pelas expressões ajustadas (expressões de [9] a [12]) que fornecem as taxas de consumo de materiais das abóbadas no domínio obtido neste estudo. Nestes gráficos, foram delimitadas regiões onde estariam as faixas de valores relatados por OLIVEIRA et al (2013), PEREIRA et al (2013) e TAVARES (2006), referentes às taxas de consumo de estruturas em concreto armado aporticadas. Esta comparação tem por objetivo de verificar as diferenças nas taxas do consumo de materiais entre coberturas em abóbadas em concreto armado e edificações aporticadas.

Figura 9 - Energia incorporada das abóbadas estudadas (fck=30 MPa)

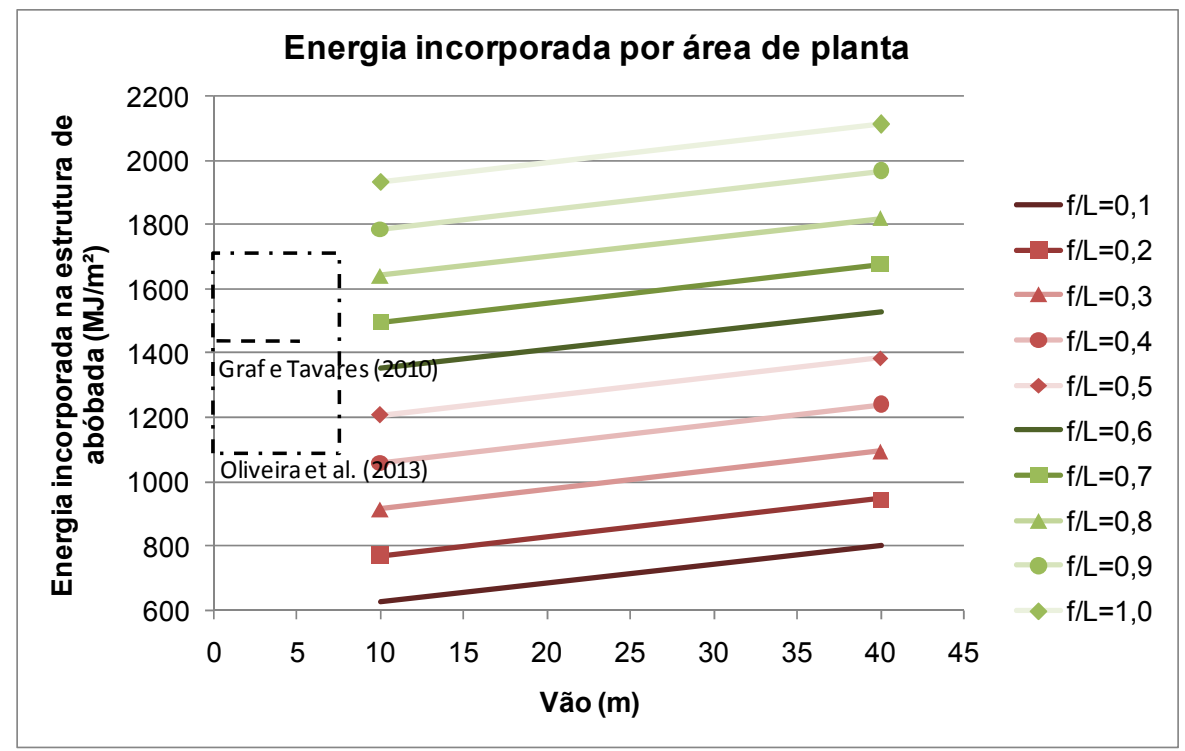

Fonte: preparada pelos autores 
Figura 10 - Carbono incorporado nas abóbadas estudadas (fck=30 MPa)

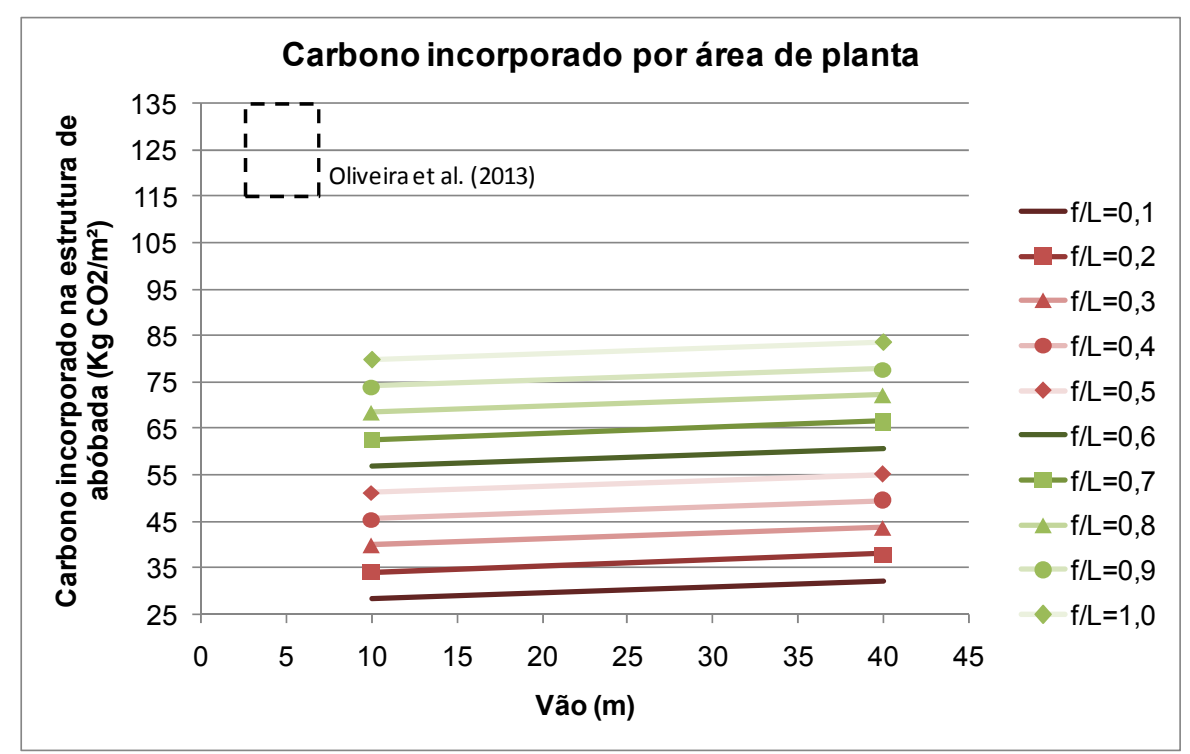

Fonte: preparada pelos autores

De maneira análoga às taxas de consumo, as Figuras 9 e 10 ilustram a energia e o carbono incorporado às abóbadas em concreto armado aqui estudadas e comparadas com as faixas de valores relatadas por GRAF e TAVARES (2010), referente a uma edificação térrea de pequeno porte, e OLIVEIRA et al. (2013), referente a edificações aporticadas em concreto de múltiplos pavimentos. Para a obtenção destes valores de energia e carbono incorporados referentes às abóbadas, foram considerados os valores de referências tanto para o concreto quanto para o aço de TAVARES (2006).

\section{CONCLUSÕES}

Diferentemente dos resultados apresentados em REBELO (2011) e CORKILL (1969), neste trabalho foram obtidas expressões analíticas para o pré-dimensionamento da abóbada em casca de concreto partindo de uma formulação simplificada. Também foram obtidas expressões ajustadas sobre os dados obtidos de protótipos modulares gerados através destas equações de pré-dimensionamento.

Evidentemente que as expressões não são práticas para serem utilizadas pelos projetistas como se utilizam os ábacos e percentuais de pré-dimensionamento já publicados na literatura, entretanto podem ser utilizadas para gerarem novos ábacos ou até utilizadas em algoritmos generativos e paramétricos deste sistema estrutural.

Quando as taxas encontradas são multiplicadas pelos preços unitários dos materiais no mercado, obtemos os custos resultantes do uso destes materiais. De outra forma, quando multiplicamos estas mesmas taxas pelos índices de energia incorporada e de geração de $\mathrm{CO}_{2}$, obtemos valores do impacto ambiental. Estes índices de recursos financeiros para construção ou do impacto ambiental do edifício, disponíveis em fases iniciais da concepção e projeto deste sistema estrutural facilitará o processo de determinação de qual é o cenário ótimo para aplicação deste sistema.

\section{AGRADECIMENTOS}

Os autores agradecem à Fundação de Amparo à Pesquisa do Estado da Bahia - FAPESB, pela assistência financeira prestada para a realização deste trabalho, e esperamos que seja de grande valia para os futuros trabalhos com ele relacionados. 


\section{REFERÊNCIAS}

ASSOCIAÇÃO BRASILEIRA DE NORMAS TÉCNICAS. NBR 6118. Projetos de estruturas de concreto - Procedimento. Rio de Janeiro,2003.

CORKILL, P. A. Preliminary Structural Design Charts for Architects. Architectural Science Review, 12:1, 15-20, 1969.

GRAF, H. F.; TAVARES, S. F. Energia incorporada dos materiais de uma edificação padrão brasileira residencial. In: $I^{\circ}$ CONGRESSO DE INOVAÇÃO, TECNOLOGIA E SUSTENTABILIDADE, 2010. Brusque-SC, 2010. p. 7.

ITEC - INSTITUTO DE TECNOLOGÍA DE LA CONSTRUCCÍON. Base de dados BEDEC. Disponível em:<http://itec.es/nouBedec.e/bedec.aspx> Acessado em: 19 maio 2015.

OLIVEIRA, F. R. M.; SILVA, M. G.; GOMES, V. Indicadores de desempenho funcional, ambiental e econômico de sistemas de lajes em concreto. $55^{\circ}$ Congresso Brasileiro do Concreto, 2013.

PEREIRA, R. L.; VINHAIS, G. C.; ARAÚJO, Y. R. O. Análise de indicadores de projetos estruturais de edifícios de múltiplos pavimentos. $55^{\circ}$ Congresso Brasileiro do Concreto, 2013.

REBELO, Yopanan C. P. Bases para o projeto estrutural na arquitetura. 3 ed. São Paulo, Zigurate Editora, 2011.

TAVARES, S. F. Metodologia de análise do ciclo de vida energético de edificações residenciais brasileiras. Tese de doutorado do programa de pós graduação em engenharia civil. UFSC, 2006. 